\title{
Design and Optimization of Feedforward-PID Integrated Controller for Pre-Injection Numerical Control Air-Powered Engine
}

\author{
Zhao Caihong $^{1, *}$, Chen Shian ${ }^{2}$ and Zhang Shijin ${ }^{2}$ \\ ${ }^{I}$ Nanhang Jincheng College, Nanjing 211156, P.R. China \\ ${ }^{2}$ School of Automotive and Traffic Engineering, Jiangsu University, Zhenjiang, P.R. China
}

\begin{abstract}
A feedforward-PID integrated controller is developed and optimized for the pre-injection numerical control (NC) air-powered engine (PINCAPE) to track the expected speed quickly and accurately. The proposed integrated controller comprises two parts, namely a feedforward controller and a PID one. According to the real-time workload and the expected speed, the feedforward controller uses a numerical fitting function to determine the main part of the supply air pressure to obtain good stable accuracy. The function is developed with the output of the supply air pressure, and the inputs of the engine speed and the output power, which are based on the different steady-state operations of the engine. To improve the engine response performance, the PID controller gives the fine-tuning of the supply air pressure with the input of the difference between the expected speed and the real-time speed. The parameters of the PID controller are optimized according to the genetic algorithm, with an objective function that minimizes the mean square of the speed error. The results show that the optimized feedforward-PID integrated controller shortens the response time of the PINCAPE by more than $47 \%$ and $64.5 \%$ under varied set point speed and engine load variations respectively, compared with the single feedforward controller. Furthermore, the response steady state error of the feedforward-PID integrated controller is less than $1 \%$, which perfectly satisfies the control specification.
\end{abstract}

Keywords: Air-powered engine, feedforward-PID integrated controller, genetic algorithm, NC, pre-injection.

\section{INTRODUCTION}

Two major problems that are facing our world today are the environment pollution and the energy crisis. The environment pollution and the energy crisis are due to more vehicles, most of which are powered by internal combustion engines using fossil fuel $[1,2]$.

The air-powered engine can give renewable power and zero emission because that is driven by compressed air which can be produced from solar, wind energy, water energy and so on [3-7]. Therefore the air-powered engine becomes more popular and is regarded as one of the ideal power sources for future vehicles.

Although there is an air-powered swash plate engine reported [8], most of air-powered engines resemble to common internal combustion engines. The air-powered engine at least has a cylinder, a piston, a crank, a valve train system, and so on. During the air-powered engine operation, there are working stroke and exhaust stroke. With the help of the valve train system, the working stroke puts compressed air into the cylinder and expands. The high pressure air expansion gives power by the piston and the crank motion. Exhaust stroke gets rid of expanded pressure air from the cylinder.

*Address correspondence to this author at the School of Mechanical Engineering, Nanhang Jincheng College, Nanjing, Jiangsu, 211156, P.R. China; Tel: +8625 87190150; E-mail: 7766733@qq.com
Air intake and exhaust of the air-powered engine are through by intake and exhaust valves of the valve train system. Because intake air pressure is much larger than the atmospheric pressure, there are difficulties in seals of the valve train system. To settle down these difficulties, a pre-injection numerical control air-powered engine (PINCAPE) is firstly given by the authors [9].

Fig. (1) shows the working scheme of the PINCAPE. The PINCAPE is at least made of a third high-pressure air tank, a pre-injection cylinder, solenoid valves, sensors and an ontology engine, without a valve timing mechanism. The preinjection cylinder and the controlled solenoid valves are set to perform the functions of the valve train system, so these seal matters in the valve train system are expected to be settled down and the energy (mass of compressed air) to the PINCAPE can be precisely provided according to its power requirement.

Chen et al. presented respective influences of intake advance angle, basal of intake sustain angle, exhaust advance angle and exhaust delay angle on the energy conversion efficiency and the output torque of the air-powered engine [10]. However, their model was not dynamic since it is based on the angular coordinate without time variation. The paper developed a non-linear model of the PINCAPE that fully displayed inner thermodynamic characteristics. 


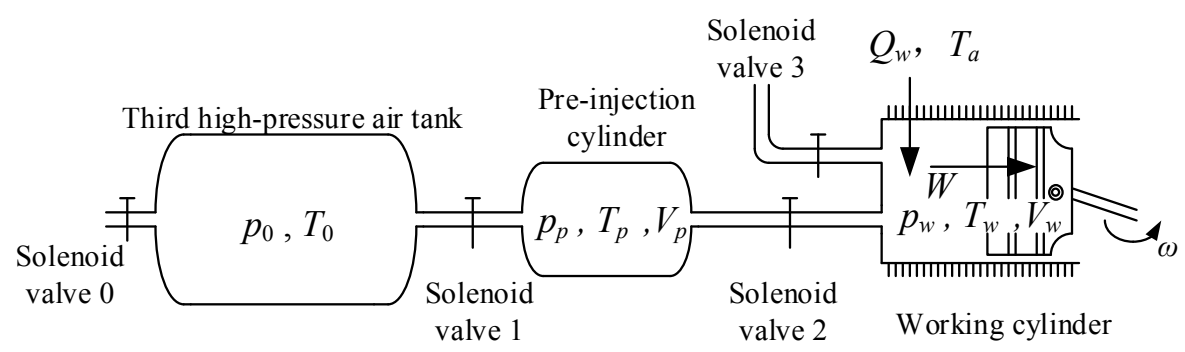

Fig. (1). working scheme of PINCAPE.

The engine with good performances should respond quickly and precisely to the speed operation. The speed control method of the existing air-powered engine with a valve train system is similar to the common internal combustion engine. The current method uses a governor to change the effective flow area of air intake to the cylinder to control the output power (or torque). Although the mechanical governor replaced by an electronic governor, the control accuracy and energy efficiency is not ideal [11]. The reason is that the control procedure is synchronous with the air intake process.

The PINCAPE has a characteristic that the amount of compressed air intake is prepared in advance of compressed air intake of the working cylinder. Hence, higher control accuracy and higher energy efficiency is expected to reach when a good speed control controller is adopted for the PINCAPE.

To improve response rate and control accuracy to the expected speed of the speed operation, the paper proposes and optimizes a feedforward-PID integrated controller for PINCAPE. The feedforward-PID integrated controller consists of a feedforward controller and a PID controller. The feedforward controller plays the role to decide the main supply pressure of the pre-injection cylinder to obtain good stable accuracy. The PID controller plays the role to decide the fine tuning supply pressure to fasten the engine's response rate. To achieve a good control effect, the PID control parameters are optimized according to the genetic algorithm (GA) $[12,13]$.

\section{THERMO-DYNAMICS MODEL OF PINCAPE}

As shown in Fig. (1), during the working stroke, the solenoid valve 2 opens, after compressed air flows into the working cylinder from the pre-injection cylinder to drive the piston and the crank, the ontology engine supplies power by the flywheel.

There are those basic hypotheses [10]:

(1) The high-pressure air is ideal gas.

(2) $p_{0}$ and $T_{0}$ are constants which are the absolute pressure and the absolute temperature in the third high-pressure air tank respectively.

(3) Equal pressure and equal temperature are throughout the pre-injection cylinder and the working cylinder.

Control strategies of the solenoid valves 1, 2 and 3 are arranged as follows.

(1) The solenoid valve 1 opens at the start of the exhaust stroke and closes when the expected control pressure of the pre-injection cylinder $p_{p e c}$ is reached.

(2) The solenoid valve 2 just opens during the working stroke.

(3) The solenoid valve 3 opens during the exhaust stroke and when the absolute pressure in the working cylinder $p_{w}$ is larger than the atmospheric pressure $p_{a}$.

$K_{1}, K_{2}$ and $K_{3}$, the switching values of the solenoid valves 1,2 and 3 , are expressed as.

$\left\{\begin{array}{l}K_{1}=\operatorname{hardlim}\left(p_{p e c}-p_{p}\right) \times\left(1-K_{2}\right) \\ K_{2}=\operatorname{hardlim}[\sin (\omega t)] \\ K_{3}=\operatorname{hardlim}\left(p_{w}-p_{a}\right) \times\left(1-K_{2}\right)\end{array}\right.$

Where the hardlim $(\cdot)$ is the hard-limit transfer function, $\omega$ is the engine speed with the unit of $\mathrm{rad} / \mathrm{s}, t$ is time.

\subsection{Thermo-Flow Rate Model of Pre-Injection Cylinder}

During intake and exhaust of the pre-injection cylinder, there are thermodynamics equations as follows [14].

$$
\begin{aligned}
& \frac{\mathrm{d} U_{p}}{\mathrm{~d} t}=K_{1} \times \frac{\mathrm{d} H_{1}}{\mathrm{~d} t}-K_{2} \times \frac{\mathrm{d} H_{2}}{\mathrm{~d} t} \\
& \frac{\mathrm{d} U_{p}}{\mathrm{~d} t}=\frac{\mathrm{d}\left(m_{p} u_{p}\right)}{\mathrm{d} t}=\frac{\mathrm{d}\left(m_{p} c_{V} T_{p}\right)}{\mathrm{d} t} \\
& p_{p} V_{p}=m_{p} R T_{p} \\
& \frac{\mathrm{d} H_{1}}{\mathrm{~d} t}=h_{0} \frac{\mathrm{d} m_{1}}{\mathrm{~d} t}=c_{p} T_{0} \frac{\mathrm{d} m_{1}}{\mathrm{~d} t}=c_{p} T_{0} q_{1} \\
& \frac{\mathrm{d} H_{2}}{\mathrm{~d} t}=h_{p} \mathrm{~d} m_{2}=c_{p} T_{p} \mathrm{~d} m_{2}=c_{p} T_{p} q_{2}
\end{aligned}
$$

Where $U_{p}, H_{1}$ and $H_{2}$ are the internal energy in the preinjection cylinder, the enthalpy flowing through the solenoid valve 1 and the enthalpy flowing through the solenoid valve 2 respectively; $c_{p}$ and $c_{V}$ are the specific heat capacity at constant pressure and the specific heat capacity at constant volume respectively; $m_{p}$ is the air mass within the pre-injection cylinder; $m_{1}$ and $m_{2}$ are the air mass flowing through the solenoid valve 1 and the air mass flowing through the solenoid valve 2 respectively; $h_{p}$ and $u_{p}$ are the specific enthalpy and the specific internal energy of the air in the pre-injection cylinder respectively; $h_{0}$ is the specific enthalpy of the air in the third high-pressure air tank; $R$ is the gas constant; $q_{1}$ and $q_{2}$ are the air flow through the solenoid valve 1 and the air flow through the solenoid valve 2 respectively. 
The pressure-flow equations of during intake and exhaust of the pre-injection cylinder are written as follows.

$$
\left\{\begin{array}{l}
q_{1}=A_{1} p_{0} \sqrt{\frac{2 k}{k-1} \frac{1}{R T_{0}}} \phi_{1}\left(\sigma_{1}\right), \quad \sigma_{1}=\frac{p_{p}}{p_{0}} \\
q_{2}=A_{2} p_{p} \sqrt{\frac{2 k}{k-1} \frac{1}{R T_{p}}} \phi_{2}\left(\sigma_{2}\right), \quad \sigma_{2}=\frac{p_{w}}{p_{p}}
\end{array}\right.
$$

Where $A_{1}$ and $A_{2}$ are the effective flowing area of the solenoid valve 1 and the effective flowing area of the solenoid valve 2 respectively; $k$ is the specific heat ratio; $\varphi_{1}$ and $\varphi_{2}$ are the stream functions associated with the pressure different through the solenoid valves 1 and 2 respectively.

$\varphi_{1}$ and $\varphi_{2}$ are written as follows [10].

$\phi_{i}\left(\sigma_{i}\right)=\left\{\begin{array}{l}\sqrt{\sigma_{i}^{\frac{2}{k}}-\sigma_{i}^{\frac{k+1}{k}}}, \quad \sigma_{i}>0.528 \\ \sqrt{\left(\frac{2}{k+1}\right)^{\frac{2}{k-1}}-\left(\frac{2}{k+1}\right)^{\frac{k+1}{k-1}}}, \quad \sigma_{i} \leq 0.528\end{array} \quad(i=1,2)\right.$

Take Equations (3), (4), (5) and (6) into Equation (2), $\frac{\mathrm{d} p}{\mathrm{~d} t}$ can be expressed as following.

$\frac{\mathrm{d} p_{p}}{\mathrm{~d} t}=\frac{k R T_{0}}{V_{p}}\left(K_{1} \times q_{1}\right)-\frac{k R T_{p}}{V_{p}}\left(K_{2} \times q_{2}\right)$

\subsection{Thermo-Flow Rate Model of Working Cylinder}

During the working stroke and the exhaust stroke of the working cylinder, there are thermodynamics equations as follows.

$\frac{\mathrm{d} Q_{w}}{\mathrm{~d} t}=\frac{\mathrm{d} U_{w}}{\mathrm{~d} t}-K_{2} \times \frac{\mathrm{d} H_{2}}{\mathrm{~d} t}+K_{3} \times \frac{\mathrm{d} H_{3}}{\mathrm{~d} t}+\frac{\mathrm{d} W}{\mathrm{~d} t}$

$\frac{\mathrm{d} U_{w}}{\mathrm{~d} t}=\frac{\mathrm{d}\left(m_{w} u_{w}\right)}{\mathrm{d} t}=\frac{\mathrm{d}\left(m_{w} c_{V} T_{w}\right)}{\mathrm{d} t}$

$p_{w} V_{w}=m_{w} R T_{w}$

$\frac{\mathrm{d} Q_{w}}{\mathrm{~d} t}=a_{g} A_{g}\left(T_{a}-T_{w}\right)$

$\frac{\mathrm{d} H_{3}}{\mathrm{~d} t}=h_{w} \mathrm{~d} m_{3}=c_{p} T_{w} \mathrm{~d} m_{3}=c_{p} T_{w} q_{3}$

$\frac{\mathrm{d} W}{\mathrm{~d} t}=p_{w} \frac{\mathrm{d} V_{w}}{\mathrm{~d} t}$

Where $Q_{w}, U_{w}, H_{3}$ and $W$ are the heat exchange between the air within the working cylinder and the environment, the internal energy within the working cylinder, the enthalpy flowing through the solenoid valve 3 and the power from compressed air within the working cylinder to the piston respectively; $m_{w}$ is the air mass within the working cylinder; $m_{3}$ is the air mass flowing through the solenoid valve $3 ; h_{w}$ and $u_{w}$ are the specific enthalpy and the specific internal en- ergy of the air within the working cylinder respectively; $q_{3}$ is the air flow through the solenoid valve $3 ; a_{g}$ is the heat exchange coefficient between the air within the working cylinder and the environment [15]; $A_{g}$ is the heat exchange area; $T_{a}$ and $T_{w}$ are the atmospheric temperature and the air temperature within the working cylinder respectively; $V_{w}$ is the working volume of the working cylinder.

$$
\begin{aligned}
& a_{g}=1.28 \sqrt[3]{\omega} \sqrt{\frac{p_{w}}{10^{5}} T_{w}}=0.004 \sqrt[3]{\omega} \sqrt{p_{w} T_{w}} \\
& q_{3}=A_{3} p_{w} \sqrt{\frac{2 k}{k-1} \frac{1}{R T_{w}}} \phi_{3}\left(\sigma_{3}\right), \quad \sigma_{3}=\frac{p_{a}}{p_{w}} \\
& \phi_{3}\left(\sigma_{3}\right)= \begin{cases}\sqrt{\sigma_{3}^{\frac{2}{k}}-\sigma_{3}^{\frac{k+1}{k}}} \quad \sigma_{3}>0.528 \\
\sqrt{\left(\frac{2}{k+1}\right)^{\frac{2}{k-1}}-\left(\frac{2}{k+1}\right)^{\frac{k+1}{k-1}}} \quad \sigma_{3} \leq 0.528\end{cases}
\end{aligned}
$$

$\mathrm{V}_{\mathrm{w}}, \mathrm{A}_{\mathrm{g}}, \frac{\mathrm{d} V_{w}}{\mathrm{~d} t}$ and $\mathrm{a}_{\mathrm{g}}$ are written as follows $[16,17]$.

$V_{w}=V_{w 0}+\frac{V_{h}}{2}\left(1-\cos \varphi+\frac{\lambda}{2} \sin ^{2} \varphi\right)$

$V_{h}=2 r S$

$S=\frac{\pi D^{2}}{2}$

$A_{g}=2 S+\pi D r\left(1-\cos \varphi+\frac{\lambda \sin ^{2} \varphi}{2}\right)$

$\frac{\mathrm{d} V_{w}}{\mathrm{~d} t}=\frac{\omega V_{h}}{2}\left(\sin \varphi+\frac{\lambda}{2} \sin 2 \varphi\right)$

$\omega=\dot{\varphi}$

Where $A_{3}$ is the effective flowing area of the solenoid valve $3 ; V_{w 0}$ is the minimum of $V_{w} ; V_{h}$ is the displacement of the working cylinder; $\varphi$ is the engine displacement; $r$ is the radius of the crank; $\lambda$ is the connecting rod ratio; $D$ is the diameter of the working cylinder; $S$ is the cross-sectional area of the working cylinder respectively.

Takeing Equations (11), (12), (13), (14), (15) and (19) into Equation (10), $\frac{\mathrm{d} p_{w}}{\mathrm{~d} t}$ can be expressed as following.

$$
\begin{aligned}
\frac{\mathrm{d} p_{w}}{\mathrm{~d} t}= & \frac{R a_{g} A_{g}}{c_{V} V_{w}}\left(T_{a}-T_{w}\right)+\frac{k R T_{p}}{V_{w}}\left(K_{2} \times q_{2}\right) \\
& -\frac{k R T_{w}}{V_{w}}\left(K_{3} \times q_{3}\right)-\frac{k p_{w}}{V_{w}} \frac{\mathrm{d} V_{w}}{\mathrm{~d} t}
\end{aligned}
$$

\subsection{Dynamics Model of Ontology Engine}

When kinematics analyses and dynamics analyses on the ontology engine are carried out, following equations are obtained. 


$$
\begin{aligned}
M_{p}= & r\left[\left(p_{w}-p_{a}\right) S-m_{i} r \omega^{2}(\cos \varphi+\lambda \cos 2 \varphi)\right] \\
& \times\left(\sin \varphi+\frac{\lambda \sin 2 \varphi}{2 \sqrt{1-\lambda^{2} \sin ^{2} \varphi}}\right)
\end{aligned}
$$

$M_{p}-M_{l}=I_{f} \ddot{\varphi}$

Where $M_{p}$ and $M_{l}$ are the torque output of the piston and the resisting torque of the engine load respectively; $I_{f}$ is the moment of inertia on the flywheel; $m_{i}$ is the reciprocating mass in the working cylinder.

$M_{l}$ is given as follows [18].

$$
\left\{\begin{array}{l}
M_{l}=M_{l 0} \operatorname{sign}(\omega)+k_{l} \omega, \quad \omega \neq 0 \\
-M_{l 0} \leq M_{l} \leq M_{l 0}, \quad \omega=0
\end{array}\right.
$$

Where $k_{l}$ is the linear resisting torque coefficient; $M_{l 0}$ is the coulomb damping of the engine.

\section{DESIGN OF FEEDFORWARD-PID INTEGRATED CONTROLLER FOR PINCAPE}

The design and optimization of the feedforward-PID integrated controller for the PINCAPE are employed by numerical simulations.

The PINCAPE is reformed from an S195 engine, the parameter values in simulations are listed in Table $\mathbf{1}$.

Table 1. Parameters in simulation.

\begin{tabular}{|c|c|c|c|}
\hline Parameter & Value & Parameter & Value \\
\hline \hline$A_{1} / \mathrm{m}^{2}$ & $7.854 \times 10^{-5}$ & $k$ & 1.4 \\
\hline$A_{2} / \mathrm{m}^{2}$ & $7.854 \times 10^{-5}$ & $c_{V} / \mathrm{J} \cdot \mathrm{kg}^{-1} \cdot \mathrm{K}^{-1}$ & 717.9 \\
\hline$A_{3} / \mathrm{m}^{2}$ & $1.5708 \times 10^{-4}$ & $c_{p} / \mathrm{J} \cdot \mathrm{kg}^{-1} \cdot \mathrm{K}^{-1}$ & 1005 \\
\hline$D / \mathrm{m}$ & 0.095 & $R / \mathrm{J} \cdot \mathrm{kg}^{-1} \cdot \mathrm{K}^{-1}$ & 287.1 \\
\hline$r / \mathrm{m}$ & 0.0575 & $p_{0} / \mathrm{Pa}$ & $10^{7}$ \\
\hline$\lambda$ & 0.2738 & $p_{a} / \mathrm{Pa}$ & $1.01 \times 10^{5}$ \\
\hline$V_{p} / \mathrm{m}^{3}$ & $1.3586 \times 10^{-4}$ & $p_{p 0} / \mathrm{Pa}$ & $2 \times 10^{6}$ \\
\hline$V_{w 0} / \mathrm{m}^{3}$ & $2 \times 10^{-5}$ & $p_{w 0} / \mathrm{Pa}$ & $1.01 \times 10^{5}$ \\
\hline$m_{i} / \mathrm{kg}$ & 2.325 & $T_{a} / \mathrm{K}$ & 300 \\
\hline$M_{l 0} / \mathrm{N} \cdot \mathrm{m}$ & 0.2 & $T_{p 0} / \mathrm{K}$ & 300 \\
\hline$I_{f} / \mathrm{kg} \cdot \mathrm{m}^{2}$ & 0.253 & $T_{w 0} / \mathrm{K}$ & 300 \\
\hline$\omega_{0} / \mathrm{rad} \cdot \mathrm{s}^{-1}$ & 0.2094 & & \\
\hline
\end{tabular}

$p_{p 0}, p_{w 0}, T_{p 0}, T_{w 0}$ and $\omega_{0}$ are the initial values of $p_{p}, p_{w}, T_{p}$, $T_{w}$ and $\omega$ respectively.

When $M_{l}$ is set as constant $\left(k_{l}=0.25 \mathrm{~N} \cdot \mathrm{m} \cdot \mathrm{s} \cdot \mathrm{rad}^{-1}\right), p_{p e c}$ is changed among 0.8 MPa, 1.6 MPa and $2.4 \mathrm{MPa}$, the $n_{r^{-}} t$ curves of the PINCAPE are given in Fig. (2). Here, $n_{r}$ is the real-time speed of the engine.

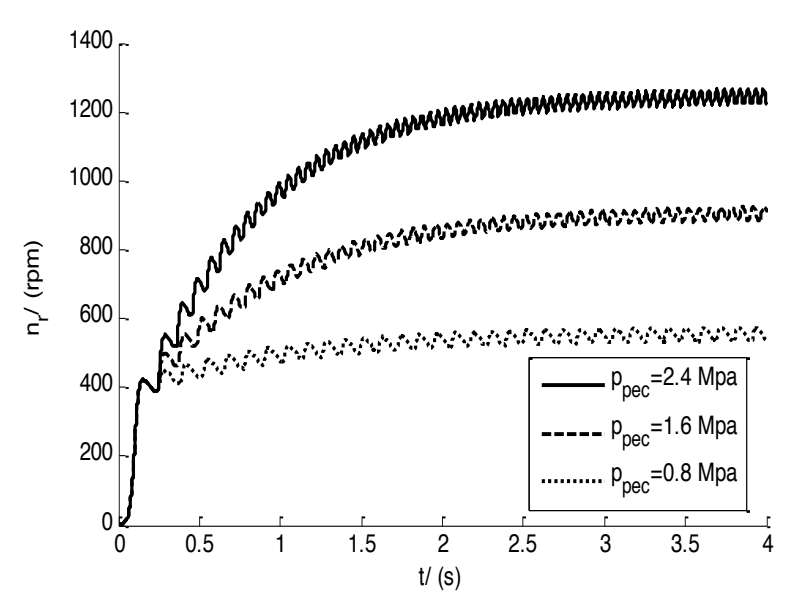

Fig. (2). $n_{r^{-}} t$ curve of PINCAPE.

Fig. (2) shows that the start-up of the PINCAPE is good because it is into working stably less than 1.5 seconds after start. The engine speed increases in air stroke and decreases in exhaust stroke when the engine is stably working and similar to common internal combustion engines. Hence, the model of PINCAPE developed in this paper is dynamic and feasible.

The vehicle equipped with a PINCAPE needs to track the expected speed quickly and accurately. A slow speed response of the engine will cause a bad vehicle dynamic performance and bring large engine speed fluctuations which lead to bad comforts. To satisfy this objective, an integrated controller with feedforward- PID approach is introduced. In a PINCAPE speed control system, the engine speed is controlled by $p_{p e c}$, the expected control pressure of the preinjection cylinder [9]. The control input is calculated from the real-time engine load $k_{l}$, the expected speed $n_{t g t}$ and the real-time engine speed $n_{r}$. $k_{l}$ and $n_{r}$ can be measured by the crank sensors. Fig. (3) shows the block diagram of the feedforward-PID integrated controller.

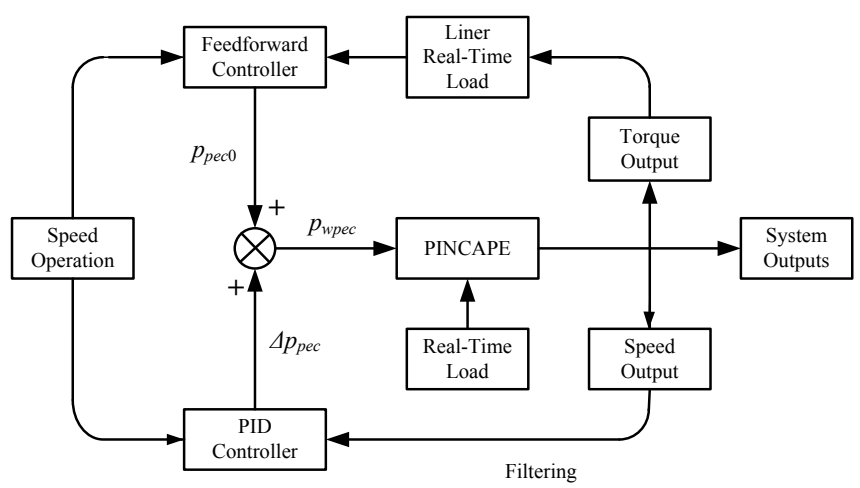

Fig. (3). block diagram of feedforward-PID integrated controller.

The feedforward-PID integrated controller gives $p_{\text {wpec }}$ which is the whole control pressure. $p_{\text {wpec }}$ is set to equal the sum of the main supply pressure of the pre-injection cylinder $p_{p e c 0}$ and the fine tuning supply pressure of the pre-injection cylinder $\Delta p_{p e c} \cdot p_{p e c 0}$ and $\Delta p_{p e c}$ are given by a feedforward loop and a PID loop respectively. 


\subsection{Feedforward Controller}

The feedforward controller gives $p_{p e c 0}$ by using $p_{p e c 0}=f\left(n_{t g t}, p_{t g t}\right)$, where the control function of $p_{p e c 0}$ is expressed by $n_{\text {tgt }}$ and $p_{\text {tgt. }} p_{\text {tgt }}$ is the output power of the engine under $k_{l}$ and $n_{\text {tgt }} . p_{\text {tgt }}$ is written as follows.

$p_{t g t}=k_{l}\left(\frac{\pi n_{t g t}}{30}\right)^{2} / 1000$

$k_{l}=\frac{M_{p r}-M_{l r}-I_{f} \dot{\omega}}{\omega+0.001}$

Where $M_{p r}$ is the measured value by the torque sensor on the crank, and $M_{l r}$ is the value of idling operation. To obtain stable $p_{t g t}, k_{l}$ is replaced by $k_{l r}$, which is filtered value of $k_{l}$ by using an Analog Filter design in Simulink [19, 20].

$p_{\text {pec } 0}=f\left(n_{t g t}, p_{t g t}\right)$ is extremely important to the feedforward controller. In order to obtain an accurate $p_{p e c 0}$, the function is established through numerical fitting method according to data of $p_{\text {spec }}, n_{s}$ and $p_{s}$, which are obtained in many different steady state engine operations. The data needed in numerical fitting are shown in Accessory 1.

$p_{\text {spec }}$ is expressed as following.

$$
\begin{aligned}
p_{s p e c}= & a_{11} n_{s}+a_{12} p_{s}+a_{21} n_{s}^{2}+a_{22} n_{s} p_{s} \\
& +a_{23} p_{s}^{2}+\mathrm{L} a_{j 1} n_{s}^{j}+\mathrm{L}+a_{j(j+1)} p_{s}^{j} \quad(j \geq 3)
\end{aligned}
$$

Where $a_{11} \sim a_{j(j+1)}$ are fitting parameters respectively. $\delta$, which is the residual between $p_{\text {spec } F}$ and $p_{\text {spec }}$, is introduced by [21].

$$
\delta=\frac{\left[\left(\sum_{i=1}^{h}\left|p_{\text {specFi }}-p_{\text {speci }}\right|\right) / h\right]}{\left[\left(\sum_{i=1}^{h}\left|p_{\text {speci }}\right|\right) / h\right]}
$$

Where $h$ is the measuring point number, $p_{\text {spec }}$ is the fitted value of $p_{\text {spec }}$.

When $j$ equals to 2, 3 and 4 respectively, the corresponding values of $\delta$ are listed in Table 2 .

Table 2. Residuals fitted of feedforward control function

\begin{tabular}{|c|c|c|c|}
\hline \multirow{2}{*}{} & \multicolumn{3}{|c|}{$j$} \\
\cline { 2 - 4 } & 2 & 3 & 4 \\
\hline$\delta$ & 0.0870 & 0.0133 & 0.0153 \\
\hline
\end{tabular}

Table 2 shows that $\delta$ is minimal when $j$ equals to 3 . Therefore, the manipulated variables of the feedforward control loop based on steady state function Equation (31) can be calculated by

$$
\begin{aligned}
p_{\text {pec } 0}= & f\left(n_{\text {tgt }}, p_{\text {tgt }}\right)=0.2778 n_{\text {tgt }}+2.9189 p_{\text {tgt }}-0.13 n_{\text {tgt }}{ }^{2} \\
& -5.3434 n_{\text {tgt }} p_{\text {tgt }}-0.0994 p_{\text {tgt }}{ }^{2}-0.0436 n_{\text {tgt }}{ }^{3} \\
& +3.3452 n_{\text {tgt }}{ }^{2} p_{\text {tgt }}+0.4572 n_{\text {tgt }} p_{\text {tgt }}{ }^{2}-0.0064 p_{\text {tgt }}{ }^{3}
\end{aligned}
$$

\subsection{PID Controller}

A standard PID controller, which is made of the proportional, the integral and derivative [22], is written as follows.

$$
\begin{aligned}
& \Delta p_{p e c}=k_{p} e+k_{i} \int_{0}^{t} e \mathrm{~d} t+k_{d} \frac{\mathrm{d} e}{\mathrm{~d} t} \\
& e=n_{t g t}-n
\end{aligned}
$$

Where $\Delta p_{p e c}$ is the fine tuning supply pressure to compensate the feedforward action to track the expected speed; $k_{p}, k_{i}$ and $k_{d}$ are the proportional, integral and derivative parameters respectively. In view that $n_{r}$ is fluctuating even if the engine works stably. To ensure the PID controller works smoothly, $n_{r}$ is replaced by $n$, which is the real-time mean value of $n_{r}$.

\section{OPTIMIZED RESULTS AND COMPARISON}

A speed operation has been applied for optimizing parameters of the designed feedforward-PID integrated controller which is that the initial engine speed was $0 \mathrm{rpm}$, in $0 \sim 15 \mathrm{~s}$ the expected speed is $900 \mathrm{rpm}$, in 15-30 s the expected speed is $1200 \mathrm{rpm}$, in $30-45 \mathrm{~s}$ the expected speed is $900 \mathrm{rpm}$, and $k_{l}$ is $0.5 \mathrm{~N} \cdot \mathrm{m} \cdot \mathrm{s} \cdot \mathrm{rad}^{-1}$.

We propose a determination method to minimize the function below.

$$
J=\int_{0}^{t} e^{2} \mathrm{~d} t
$$

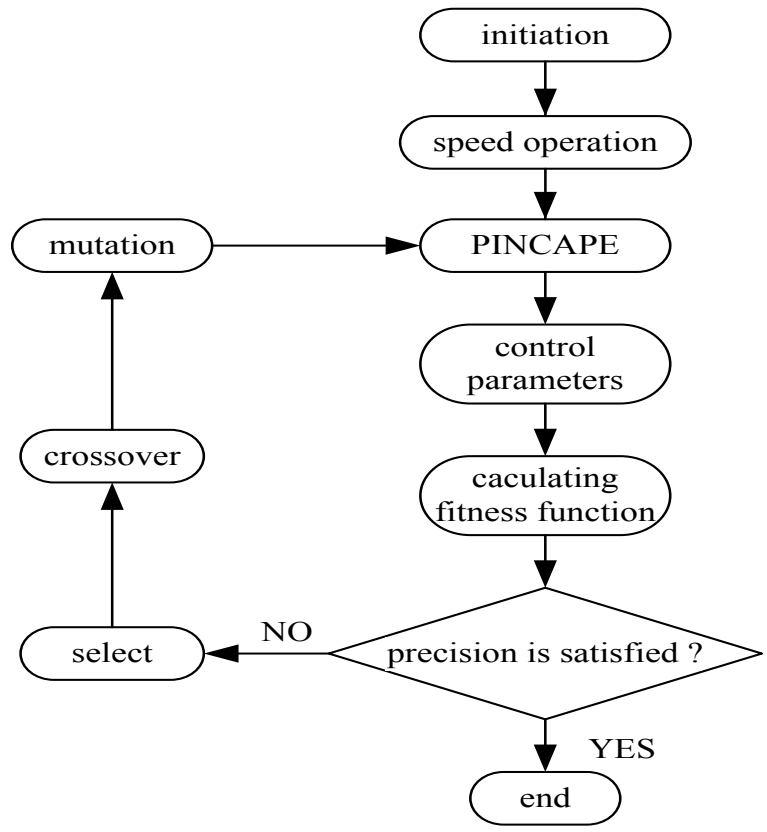

Fig. (4). Flow chart for optimizing the feedforward-PID integrated controller based on GA.

The genetic algorithm (GA) is a powerful search technique which has been applied to many practical problems. Hence, GA is chosen as the optimizing method for the feedforward-PID integrated controller [23]. During optimization procedure, GA iteratively manipulates populations of these 
chromosomes using reproduction, crossover and mutation operations, and evaluates suitability of each solution using a fitness function. Here the optimization objective function $J$ is taken as the fitness function. Chromosomes with a good fit are carried forward into the next generation and are subsequently used as the basis for generating new and improved solutions. The search procedure continues iteratively until the specified termination conditions have been satisfied. It is that the solution with desired fitness is satisfied for a specified number of generations. Flow chart for optimizing the feedforward-PID integrated controller based on GA is shown in Fig. (4).

The parameters for the GA-based optimizing procedure are selected as follows.

1) Maximum number of generation: 50;

2) Initial population size: 60 ;

3) Crossover probability: 0.9;

4) Mutation probability: 0.7;

5) Generation gap: 0.9;

6) $k_{d} \in[0,50], k_{i} \in[0,50000]$ and $k_{p} \in[0,3500]$.

Consequently, after 50 generations, $k_{d}=3.7, k_{i}=265.3$ and $k_{p}=2991.5$ are obtained, change of population optimal solution is shown in Fig. (5). The $n-t$ curves and $p_{\text {wpec }}-t$ curves are shown in Figs. $(\mathbf{6}, 7)$.

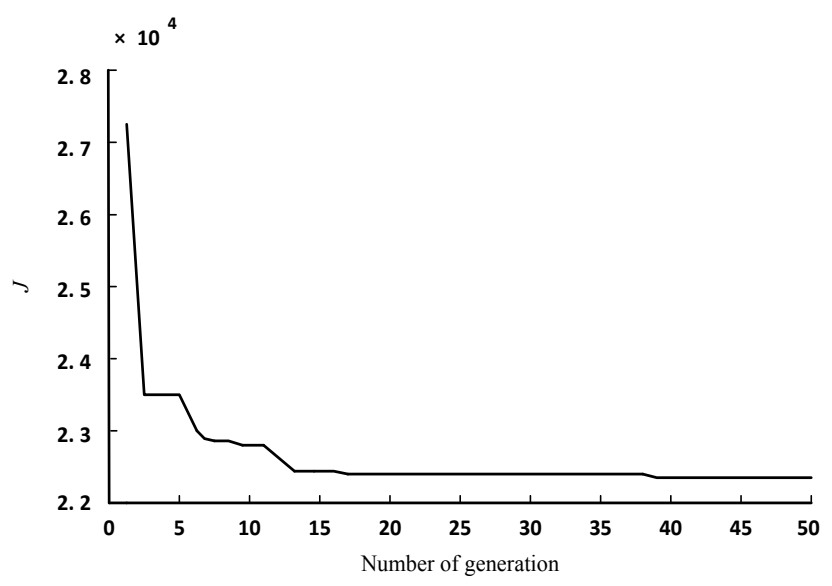

Fig. (5). Change of population optimal solution.

Fig. (6) shows the feedforward controller takes $2.42 \mathrm{~s}$ from $0 \mathrm{rpm}$ to $900 \mathrm{rpm}, 1.6 \mathrm{~s}$ from $900 \mathrm{rpm}$ to $1200 \mathrm{rpm}$, and $1.3 \mathrm{~s}$ from $1200 \mathrm{rpm}$ to $900 \mathrm{rpm}$. The corresponding times of the feedforward-PID integrated controller are $0.8 \mathrm{~s}, 0.49 \mathrm{~s}$, and $0.69 \mathrm{~s}$, respectively. Thus, the feedforward-PID integrated controller is faster than the feedforward controller. As shown in Fig. (7), the reason is that the PID controller plays useful roles to improve the control compensation performance. Fig. (6) shows that $e$ is $1 \mathrm{rpm}$ when the engine stably works at $900 \mathrm{rpm}$ and $e$ is $8 \mathrm{rpm}$ when the engine stably works at $1200 \mathrm{rpm}$. Therefore, the feedforward-PID integrated controller has an ability of tracking the speed operation accurately.

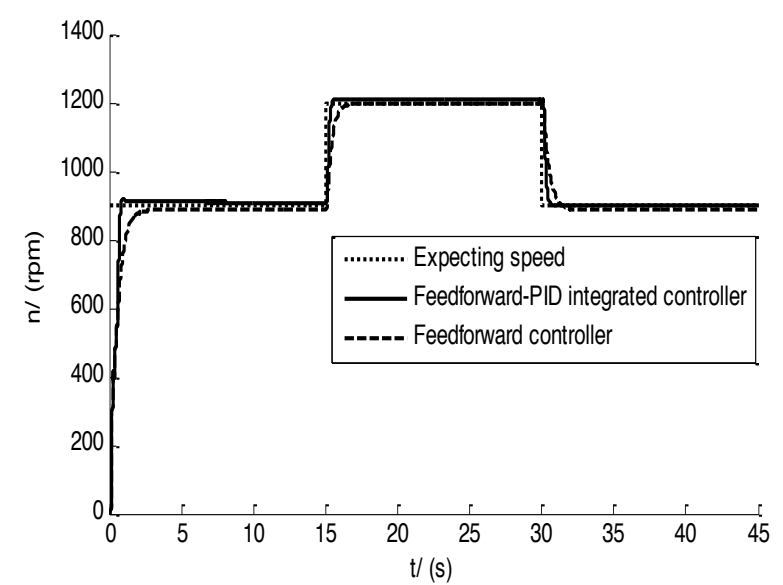

Fig. (6). $n$ - $t$ curves when $n_{t g t}$ varies.

To verify the efficacy of the feedforward-PID integrated controller, intermittent operations at load variations are simulated. The intermittent operations are that $n_{\text {tgt }}$ is fixed to $900 \mathrm{rpm}$, the $k_{l}$ was varied from $0.1 \mathrm{~N} \cdot \mathrm{m} \cdot \mathrm{s} \cdot \mathrm{rad}^{-1}$ to 1 $\mathrm{N} \cdot \mathrm{m} \cdot \mathrm{s} \cdot \mathrm{rad}^{-1}$ at the 15 th second and from $1 \mathrm{~N} \cdot \mathrm{m} \cdot \mathrm{s} \cdot \mathrm{rad}^{-1}$ to 0.1 $\mathrm{N} \cdot \mathrm{m} \cdot \mathrm{s} \cdot \mathrm{rad}^{-1}$ at the 30 th second. The $k_{l r}-t$ curve, $n$ - $t$ curves and $p_{\text {wpec }}-t$ curves are shown in Figs. (8-10).

Fig. (9). shows when $k_{l}$ varies, the feedforward controller takes $1.21 \mathrm{~s}$ and $5.8 \mathrm{~s}$ to stably reach $900 \mathrm{rpm}$. The corresponding times of the feedforward-PID integrated controller are $0.43 \mathrm{~s}$ and $1.45 \mathrm{~s}$ respectively. The reason is that the feedforward-PID integrated controller gives much bigger $p_{\text {wpec }}$ when $k_{l}$ increases and vice verse as shown in Fig. (10). Therefore, the feedforward-PID integrated controller has a good robustness.

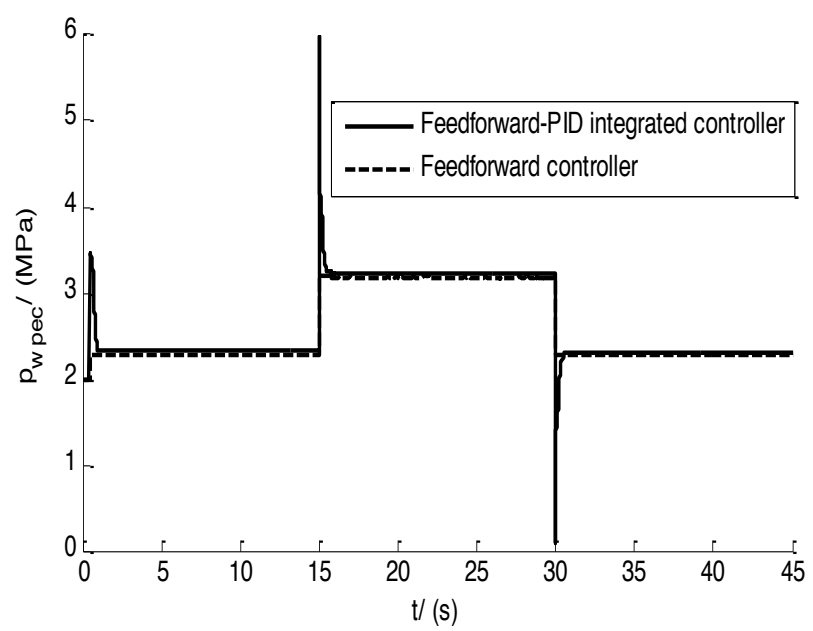

Fig. (7). $p_{\text {wpec }}-t$ curves when $n_{t g t}$ varies.

\section{CONCLUSION}

Based on the thermo-dynamics model of PINCAPE, this paper designs a robust feedforward-PID integrated controller that includes a feedforward controller and a PID controller to implement the engine speed control. For the tracking, accuracy and robustness, we compare with the feedforward con- 
troller used only and confirm the effectiveness of the proposed controller.

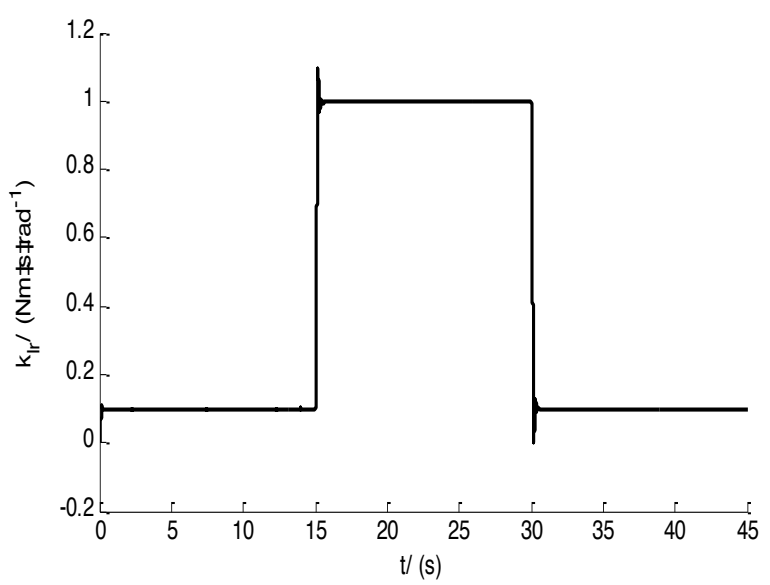

Fig. (8). $k_{l r}$ - $t$ curve.

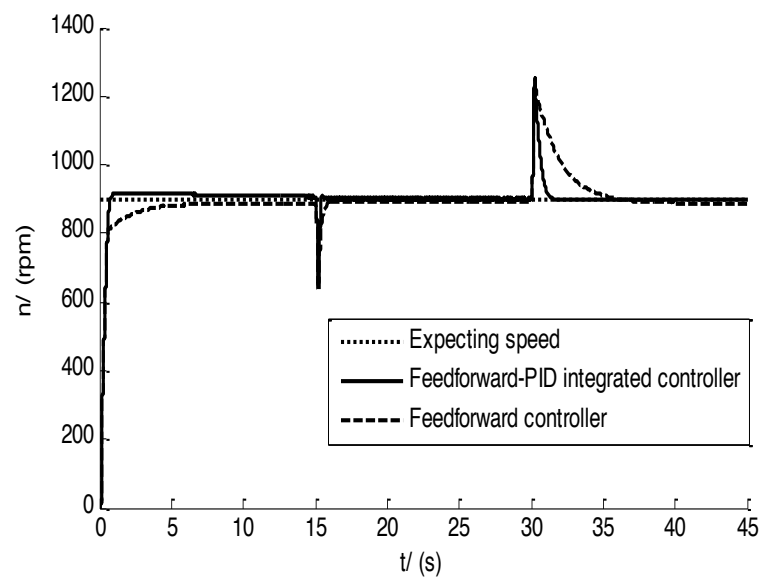

Fig. (9). $n$ - $t$ curves when $k_{l}$ varies.

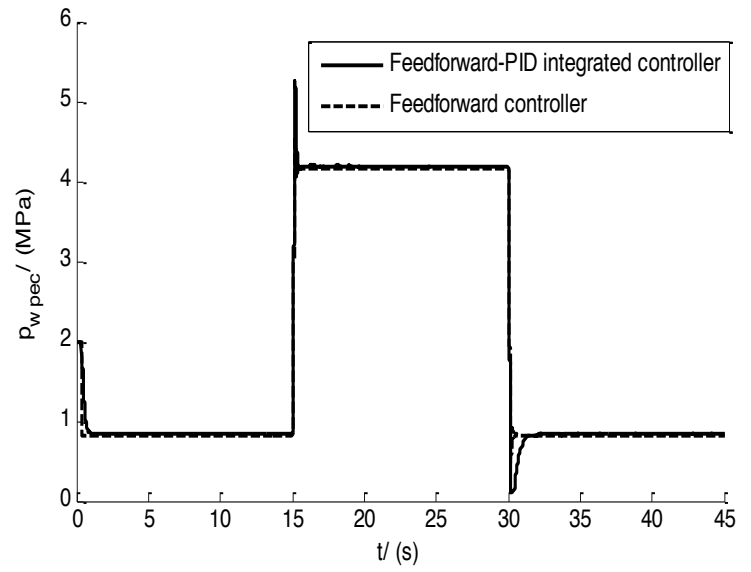

Fig. (10). $p_{\text {wpec }}-t$ curves when $k_{l}$ varies.

In the future we plan to perform experiments about a feedforward-PID integrated controller, not only verity this control method, but also offer a reference for using of control on PINCAPE.

\section{ACCESSORY 1}

All of data are recorded in Table 3
Table 3. $\quad n_{\mathrm{s}}$ and $p_{s}$ recorded in each $p_{\text {spec }}$

\begin{tabular}{|c|c|c|c|c|c|}
\hline $\begin{array}{c}\mathbf{p}_{\text {spec }} \\
\text { (MPa) }\end{array}$ & $\begin{array}{c}n_{\mathrm{s}} \\
(100 \mathrm{r} / \mathrm{min})\end{array}$ & $\begin{array}{c}\mathbf{p s}_{\mathrm{s}} \\
(\mathrm{kW})\end{array}$ & $\begin{array}{c}\mathbf{p}_{\text {spec }} \\
(\mathbf{M P a})\end{array}$ & $\begin{array}{c}n_{\mathrm{s}} \\
(100 \mathrm{r} / \mathrm{min})\end{array}$ & $\begin{array}{c}\mathbf{p}_{\mathrm{s}} \\
(\mathrm{kW})\end{array}$ \\
\hline 2 & 23.3 & 3.4 & 2 & 22.5 & 3.7 \\
\hline 2 & 21.6 & 3.9 & 2 & 21.0 & 4.1 \\
\hline 2 & 20.2 & 4.3 & 2 & 19.6 & 4.4 \\
\hline 2 & 16.9 & 4.8 & 2 & 14.8 & 4.9 \\
\hline 2 & 13.0 & 4.7 & 2 & 11.5 & 4.4 \\
\hline 2 & 10.3 & 4.1 & 2 & 9.3 & 3.8 \\
\hline 2 & 8.4 & 3.5 & 2 & 7.7 & 3.1 \\
\hline 2 & 7.1 & 3.0 & 2 & 6.5 & 2.8 \\
\hline 2 & 6.1 & 2.6 & 2 & 5.7 & 2.5 \\
\hline 3 & 22.6 & 5.7 & 3 & 20.1 & 6.8 \\
\hline 3 & 18.2 & 7.4 & 3 & 15.3 & 7.8 \\
\hline 3 & 13.2 & 7.7 & 3 & 11.4 & 7.2 \\
\hline 3 & 10.0 & 6.7 & 3 & 8.9 & 6.0 \\
\hline 3 & 7.9 & 5.5 & 3 & 7.1 & 5.0 \\
\hline 3 & 6.5 & 4.6 & 3 & 5.9 & 4.3 \\
\hline 4 & 24.2 & 6.5 & 4 & 22.0 & 8.0 \\
\hline 4 & 20.2 & 9.0 & 4 & 17.5 & 10.1 \\
\hline 4 & 15.4 & 10.5 & 4 & 13.8 & 10.4 \\
\hline 4 & 12.4 & 10.2 & 4 & 11.3 & 9.8 \\
\hline 4 & 10.2 & 9.2 & 4 & 9.4 & 8.7 \\
\hline 4 & 8.6 & 8.2 & 4 & 7.9 & 7.7 \\
\hline 5 & 23.4 & 9.0 & 5 & 21.7 & 10.4 \\
\hline 5 & 20.2 & 11.3 & 5 & 19.1 & 12.0 \\
\hline 5 & 17.1 & 12.8 & 5 & 15.4 & 13.1 \\
\hline 5 & 14.1 & 13.1 & 5 & 12.9 & 12.9 \\
\hline 5 & 12.0 & 12.6 & 5 & 11.1 & 12.2 \\
\hline 5 & 10.3 & 11.7 & 5 & 9.6 & 11.2 \\
\hline 5 & 9.0 & 10.7 & 5 & 8.4 & 10.2 \\
\hline 5 & 7.9 & 9.7 & 5 & 7.4 & 9.3 \\
\hline 5 & 7.0 & 8.8 & 5 & 6.6 & 8.4 \\
\hline 5 & 6.3 & 8.0 & 5 & 5.9 & 7.6 \\
\hline
\end{tabular}

\section{CONFLICT OF INTEREST}

The authors confirm that this article content has no conflict of interest. 


\section{ACKNOWLEDGEMENTS}

This research is financially supported by the National Natural Science Fund Project of China under the contract 50805066 and the Scientific Research Foundation for Talented Scholars of Jiangsu University under the contract 06jdg040.

\section{REFERENCES}

[1] L.G. Liu, "Development and research of the automobile in the $21^{\text {st }}$ century", Chinese Journal of Automobile Research and Development, no. 1, pp. 8-10, 2001.

[2] Y. Z. Bian, "Automobile New Energy Technology", Beijing: People's Traffic Press, 2003.

[3] J. Williams, C. Knowlen, A. T, Mattick and A. Hertzberg, "Quasi Isothermal Expansion Engines For Liquid Nitrogen Automotive Propulsion", SAE Paper, no. 972649, 1997.

[4] H. Liu, Y. Chen and G.L. Tao, "Researches on characteristics and model of work process of compressed air powered engine", Progress in Natural Science, no. 14, pp. 319-324, 2004.

[5] P.L. Chen, X.L. Yu and L. Liu, "Simulation and experimental study of electro-pneumatic valve used in air-powered engine", Journal of Zhejiang University (Science A), no. 10, pp. 377-383, 2009.

[6] H.G. Liu, B.B. Gu, F. Qiu and S.A. Chen, "Testing and Numerical Fitting of Cornering Stiffness of 7.50R20 14PR PW02Tire", Tractor \& Farm Transporter, no. 36, pp. 63-65, 2009.

[7] S.A. Chen, R He, S.L.. Lu and M. Yao, "Modeling of air-powered engine based on time coordinate and optimal approach of gas distribution parameters", Proceedings of the ASME International Design Engineering Technical Conference \& Computers and Information in Engineering Conference, IDETC/CIE2010, no. 4, pp. 219-227, 2010.

[8] Y.Q. Lian, S.Z. Wang, S.J. Ma, Y.H. Chen and Z.J. Li, "Dynamic simulation of the air-powered swash plate engine", Chinese Journal of Mechanical Engineering, no. 44, pp. 243-248, 2008.
[9] S.A. Chen, S.J. Zhang and R. He, "Operating properties of preinjection NC air-powered engine", Journal of Traffic and Transportation Engineering, no. 1, pp. 35-41, 2011.

[10] Y. Chen, H. Liu and G.L. Tao, "Simulation on the port timing of an air-powered engine", International Journal of Vehicle Design, no. 38 , pp. 259-273, 2005.

[11] S. Umerujan, T. Gheyret, T. Buso and T. Teruo, "Speed control of general-purpose engine with electronic governor", Electrical Engineering in Japan, no. 2, pp. 64-71, 2012.

[12] Y.Zhang, T. and P.G. Mehta, "Feedforward and feedback adaptive control design and analyses", Proceedings of $14^{\text {th }}$ IFAC World Congress, pp. 409-414, 1999.

[13] Y.H. Tao, "New Type of PID Control and its Application", Beijing: Machinery Industry Press, no. 3, 1998, pp. 286-297.

[14] B.D. Wood, "Applications of Thermodynamics", Massachusetts: Addison-Wesley Publishing Company, 1982, pp. 286-297.

[15] R.S. Lu, "Heat Transfer and Thermal Loading in Internal Combustion Engine”, Beijing: People's Traffic Press, 1988.

[16] J.B. Heywood, "Internal Combustion Engine Fundamentals", New York: McGraw-Hill Inc, 1998.

[17] W. Xu, "The Modern Design Of Automobile Engine", Beijing: People's Traffic Press, 1995.

[18] S. Menon. N. Moulton and C. Cadou "Development of a dynamometer for measuring small internal-combustion engine performance", Journal of Propulsion and Power, no. 23, pp. 194-202, 2007.

[19] D.Y. Xue and Y.Q. Chen "Technology and Application of System Simulation Based on MATLAB/Simulink", Beijing: Tsinghua University Press, 2002.

[20] B.Z. Yu, “Digital Signal Processing”, Xi'an: Northwestern Polytechnical University Press, 2002.

[21] L. Liu, X.L. Yu, J.Q. Hu and P.L. Chen, "Air powered engine design based on Pareto Frontier", Journal of Zhejiang University (Engineering Science), no. 43, pp. 123-127, 2009.

[22] H.X. Wu and S.P. Shen, "Basis of theory and applications on PID control", Control Engineering of China, no. 10, pp. 37-42, 2003.

[23] Y.J. Lei and S.W. Zhang, "Genetic Algorithm Tools and Applications", Xi'an: Xi'an Electronic Science \& Technology University Press, 2005.

\footnotetext{
Received: May 26, 2015

(C) Caihong et al.; Licensee Bentham Open.
}

Revised: July 14, 2015

Accepted: August 10, 2015

This is an open access articles licensed under the terms of the Creative Commons Attribution-Non-Commercial 4.0 International Public License (CC BY-NC 4.0) (https://creativecommons.org/licenses/by-nc/4.0/legalcode), which permits unrestricted, non-commercial use, distribution and reproduction in any medium, provided that the work is properly cited. 